Joseph D. Tobias MD, ${ }^{*}+\$$

Randall S. Burd MD, ${ }^{*} \neq \mathbb{I}$

Mary Alice Helikson $\mathrm{MD}^{\star} \mp \mathbb{I}$

\title{
Apnea following spinal anaesthesia in two for- mer pre-term infants
}

Purpose: To report the occurrence of apnea and bradycardia in two former pre-term infants who received spinal anaesthesia without inhalational or intravenous anaesthetic agents during inguinal herniorrhaphy.

Clinical features: Two former pre-term infants who had no recent history of apnea or bradycardia and who had been discharged from the hospital presented for anaesthetic care during inguinal herniorrhaphy. Spinal anaesthesia using I mg. $\mathrm{kg}^{-1}$ tetracaine in dextrose $10 \%$ was performed. A sensory level of $\mathrm{T}_{4-6}$ was obtained in both infants. Neither infant received intravenous, oral, or inhalational agents for sedation. Five to ten minutes after placement of the spinal block, the two infants had frequent episodes of apnea and bradycardia. No change in the sensory level of spinal anaesthesia was noted. The apnea and bradycardia continued for a variable time into the postoperative period. Caffeine benzoate $10 \mathrm{mg} \cdot \mathrm{kg}^{-1}$ was administered to one infant. Other than the apnea and bradycardia, both infants had uncomplicated postoperative courses.

Conclusion: Apnea can occur when spinal anaesthesia is used as the sole anaesthetic technique in the former pre-term infant. Appropriate monitoring for such problems based on the infant's post-conceptual age is suggested.

Objectif : Rapporter l'occurrence d'apnée et de bradycardie chez deux enfants nés prématurément qui ont reçu une rachianesthésie sans agents intraveineux ou d'inhalation pendant une herniorraphie inguinale.

Aspects cliniques : Deux enfants nés prématurément, sans histoire récente d'apnée ou de bradycardie et qui avaient reçu leur congé de l'hôpital, se sont présentés pour une herniorraphie inguinale sous anesthésie. La rachianesthésie a été réalisée avec $1 \mathrm{mg} \cdot \mathrm{kg}^{-1}$ de tétracaïne dans du dextrose $10 \%$. Un niveau sensitif à $\mathrm{T}_{4.6}$ a été obtenu chez les deux enfants. Aucun n'a reçu de sédation par voie intraveineuse ou orale ou par inhalation. Cinq à dix minutes après le blocage rachidien, les deux enfants ont présenté de fréquents épisodes d'apnée et de bradycardie. On n'a noté aucun changement du niveau sensitif de l'anesthésie rachidienne. Les manifestations d'apnée et de bradycardie, d'une durée variable, se sont poursuivies apres la chirurgie. On a administré $10 \mathrm{mg} \cdot \mathrm{kg}^{-}$ I de benzo ate de caféine à l'un des enfants. Mis à part l'apnée et la bradycardie, aucune autre complication postopératoire ne s'est présentée.

Conclusion : L'apnée peut survenir lorsqu'une rachianesthésie est la seule technique d'anesthésie utilisée chez un enfant né avant terme. Un monitorage approprié à la situation, basé sur l'âge post-conception de l'enfant, est donc recommandé.

From the Departments of Child Health, ${ }^{*}$ Anesthesiology, $\uparrow$ and Surgery $\ddagger$ and the Divisions of Pediatric Critical Care/Pediatric Anesthesiology $\$$ and Pediatric Surgery, 1 The University of Missouri, Columbia, Missouri.

Address correspondence to: Joseph D. Tobias MD, The University of Missouri, Department of Child Health, M658 Health Sciences Center, One Hospital Drive, Columbia, Missouri 65212. Phone: 573-882-6544; Fax: 573-882-2742; E-mail:

Joseph_Tobias@muccmail.missouri.edu

Accepted for publication August 7, 1998 


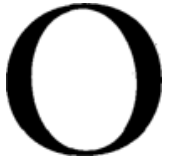

$\mathrm{NE}$ of the major concerns in the former pre-term infant is postoperative apnea and bradycardia with a reported incidence as high as $30-40 \%,{ }^{1,2}$ Spinal anaesthesia has been suggested as an alternative to general anaesthesia as a means of decreasing or eliminating the occurrence of postoperative apnea. ${ }^{3-5}$ Abajian $e t$ al. reported their experience with spinal anaesthesia in 78 infants, 36 of whom were high-risk former pre-term infants < $60 \mathrm{wk}$ postconception age. ${ }^{3}$ Their success with no cases of postoperative apnea suggested the advantages of spinal over general anaesthesia in these patients.

Other investigators reported similar degrees of success. Sartorelli et al. reported their experience with spinal anaesthesia in 140 former pre-term infants. ${ }^{4}$ They noted apnea in only one infant who had received a dose of midazolam $i v$ for sedation during the procedure. Veverka $e t$ al. noted no apnea in 84 high-risk infants following spinal anaesthesia and suggested that supplementation with intravenous ketamine for intraoperative sedation added no increased risk of apnea and that these procedures could be performed in the outpatient setting. ${ }^{5}$

Clinical experience suggests the utility and safety of spinal anaesthesia in the former pre-term infant. Despite this safety record and its suggested advantage over general anaesthesia, apnea can still occur following spinal anaesthesia. We present two former preterm infants, who had been discharged home, neither of whom had a recent history of apnea, but who developed serious apnea and bradycardia following spinal anaesthesia. Although both infants had a remote history of apnea and bradycardia, neither had had recent problems. Neither infant received intravenous sedation or inhalation anaesthetic agents.

\section{Case reports}

\section{Case \#1}

A $2 \mathrm{~kg}, 8 \mathrm{wk}$, former $30 \mathrm{wk}$ pre-term infant girl (postconceptional age $=38 \mathrm{wk}$ ) presented for repair of a right inguinal hernia with left sided inguinal exploration. The patient had a history of mechanical lung ventilation for five days for mild hyaline membrane disease and intermittent apnea and bradycardia. The infant had been discharged home at six weeks of age having had no apnea and bradycardia for four weeks. She had done well at home, continuing to feed well and grow as expected. She was not on a home apnea monitor and was receiving no medication. She was kept NPO for two hours. Following arrival in the operating room, an intravenous infusion of dextrose
$5 \%$ in lactated Ringers was started at a maintenance rate. During placement of spinal block and during the surgical procedure, the infant was cared for under a radiant heat warmer. Atropine $0.05 \mathrm{mg} i v$ was administered. The infant was placed in the sitting position and, using sterile procedure, lumbar puncture was performed. Following free flow of cerebrospinal fluid, $2 \mathrm{mg}$ tetracaine diluted in dextrose $10 \%$ and an epinephrine wash was injected. The epinephrine wash was prepared by drawing up $1 \mathrm{~mL}, 1 \mathrm{mg} \bullet \mathrm{mL}^{-1}$ epinephrine into a tuberculin syringe and then pushing the plunger all the way into the syringe to remove as much of the epinephrine as possible. Free flow of CSF was noted at the start and completion of the injection. The infant was placed supine. The eventual level of sensory blockade was $\mathrm{T}_{4-6}$ assessed using a standard twitch monitor set at tetanus of $50 \mathrm{~Hz}$. No change was noted in the heart rate or blood pressure. The surgical procedure was started. Five minutes after placement of spinal anaesthesia, the infant had a $20 \mathrm{sec}$ episode of apnea associated with bradycardia of 80 bpm and a decrease in oxygen saturation to the low $70 \%$ range. The infant was gently stimulated and immediately resumed a normal respiratory pattern and heart rate. The sensory level remained unchanged. The surgical procedure lasted $35 \mathrm{~min}$ and the infant was transferred to the post-anaesthesia care unit (PACU). In the PACU, the infant had several episodes of periodic breathing with respiratory pauses of 15-20 sec. There was no change in the heart rate, but the oxygen saturation decreased to $85-90 \%$. The spinal anaesthetic dissipated in two hours and there was a return of normal lower extremity motor function. After two hours, there were no further episodes of apnea and bradycardia. The infant was admitted to the inpatient ward with pulse oximetry and an apnea monitor. Eight hours postoperatively, while the infant was breastfeeding, she had an episode of apnea and bradycardia that required stimulation and supplemental oxygen administration. She was transferred to the Pediatric ICU for closer monitoring. She had no further episodes of apnea and bradycardia and was discharged home after $18 \mathrm{hr}$ of uneventful monitoring.

\section{Case \#2}

A $2.4 \mathrm{~kg}, 10 \mathrm{wk}$, former $28 \mathrm{wk}$ pre-term infant boy (post-conceptional age $=38 \mathrm{wk}$ ) presented for repair of a right inguinal hernia with left sided inguinal exploration. The patient had a history of 10 days of mechanical lung ventilation for mild hyaline membrane disease and intermittent apnea and bradycardia. The infant had been discharged home at eight weeks of age having had no apnea and bradycardia for four 
weeks. He had done well at home, continuing to feed well and grow as expected. He was not on a home apnea monitor and was receiving no medication. The infant was kept NPO for two hours. Following arrival in the operating room, an infusion of dextrose $5 \%$ in lactated Ringers was started at a maintenance rate. Atropine $0.05 \mathrm{mg} i v$ was administered. During placement of spinal block and during the surgical procedure, the infant was cared for under a radiant heat warmer. The infant was placed in the sitting position and, using a sterile procedure, lumbar puncture was performed. Following free flow of CSF, $2.4 \mathrm{mg}$ tetracaine diluted in dextrose $10 \%$ and an epinephrine wash was injected. Free flow of CSF was noted at the start and completion of the injection. The infant was placed supine. The eventual level of sensory blockade was $T_{6}$, assessed using a standard twitch monitor set at tetanus of $50 \mathrm{~Hz}$. No change was noted in heart rate or blood pressure. The surgical procedure started. Ten minutes after placement of spinal anaesthesia, the infant had 30 sec apnea associated with bradycardia to a heart rate of $60 \mathrm{bpm}$ and a decrease in oxygen saturation to $70 \%$. The infant was gently stimulated and immediately resumed a normal respiratory pattern and heart rate. The sensory level remained unchanged. Over the next $15 \mathrm{~min}$, the infant had three more episodes of apnea and bradycardia requiring stimulation. Caffeine benzoate $\left(10 \mathrm{mg} \cdot \mathrm{kg}^{-1}\right)$ iv was administered. The surgical procedure lasted $60 \mathrm{~min}$ and the infant was transferred to the PACU. The infant was observed for two hours in the PACU and had no further episodes of apnea. The spinal anaesthetic dissipated in $1.5 \mathrm{hr}$ and there was a normal return of lower extremity motor function. The infant was admitted to the inpatient ward with pulse oximetry and an apnea monitor. The remainder of the postoperative course was unremarkable and he was discharged home the next morning.

\section{Discussion}

We have presented two former pre-term infants who despite having no recent history of apnea and bradycardia developed severe and repeated episodes of apnea and bradycardia following uncomplicated spinal anaesthesia. Neither infant received supplemental analgesia or sedative agents during their perioperative care which might have contributed to the apnea and bradycardia. Immediately after the apnea and bradycardia, repeat physical examination demonstrated that the sensory level of the spinal anaesthetic remained at $\mathrm{T}_{6}$.

Spinal anaesthesia has been suggested as a means of limiting anaesthetic-related perioperative morbidity. Welborn et al. randomly assigned former pre-term infants with a postconceptional age of $51 \mathrm{wk}$ or less to receive either general or spinal anaesthesia. ${ }^{6}$ In the first part of the study, infants randomized to spinal anaesthesia received $2 \mathrm{mg} \cdot \mathrm{kg}^{-1}$ ketamine for sedation prior to the lumbar puncture. None of the patients $(n=11)$ who received spinal anaesthesia without ketamine sedation had apnea. Eight of nine infants who received spinal anaesthesia with ketamine sedation developed apnea while 5 of 16 patients who received general anaesthesia developed apnea $(P=\mathrm{NS})$. There was no difference in the incidence of apnea between general anaesthesia and spinal anaesthesia in infants without a previous history of apnea.

Krane $e t$ al. compared the incidence of postoperative apnea, oxygen saturation, and periodic breathing in 80 former pre-term infants randomized to receive either general or spinal anaesthesia. ${ }^{7}$ Infants who received general anaesthesia had differences in minimum oxygen saturation, lowest heart rate, and number of episodes of oxygen desaturation to $<80 \%$ when compared with infants who received spinal anaesthesia. There was no difference in the incidence of central apnea. Although the methods section states that infants in the spinal anaesthesia group who cried during surgical manipulation received nitrous oxide $50 \%$, there was no mention of attempting to correlate this with postoperative apnea.

The literature is confusing when attempting to determine whether previous reports demonstrate apnea and bradycardia in infants that had received only a spinal anaesthetic without intravenous or inhalation sedative/analgesic agents. It is not possible to discern from the study of Krane et al. ${ }^{7}$ whether there was apnea in infants who received spinal anaesthesia alone or only in those who received nitrous oxide plus spinal anaesthesia. Welborn et al. ${ }^{6}$ reported apnea only in infants that received intravenous ketamine to supplement the spinal block. The one infant in the study of Sartorelli $e t$ al. who developed postoperative apnea also received intravenous midazolam. ${ }^{4}$ Harnik et al. ${ }^{8}$ reported that 2 of 20 infants developed apnea following spinal anaesthesia. One of the infants had ongoing problems with apnea and bradycardia while the second infant was hypothermic. Webster $\boldsymbol{e t}$ $a l^{9}$ reported apneic events requiring bag/mask ventilation in six of 44 infants receiving spinal anaesthesia. Confounding factors in the six infants that developed apnea included one who developed apnea before any anaesthetic intervention, "high spinal anaesthesia" (level not specified) in two infants, and apnea only following intravenous ketamine in the remaining three infants.

Cox et al. ${ }^{10}$ reported two infants who developed apnea following spinal anaesthesia. Both patients 
received nitrous oxide to supplement the spinal anaesthesia during traction on the hernia sac as well as receiving oral codeine for postoperative pain management. In addition, both patients had a recent history of apnea and bradycardia and were receiving oral theophylline with therapeutic levels. Cox's second patient was also hypothermic $\left(34^{\circ} \mathrm{C}\right)$, anaemic (hacmoglobin $\left.7.1 \mathrm{~g} \cdot \mathrm{dL}^{-1}\right)$, and subsequently found to be bacteraemic.

Watcha et al. reported a case similar to ours in a 3.9 $\mathrm{kg}, 4$ mo old ( $42 \mathrm{wk}$ postconception) infant who had no recent history of apnea, had been home for one month, and was receiving no medication. ${ }^{11} \mathrm{~A}$ caudal epidural block was placed for a herniorrhaphy without supplementation with intravenous or inhalation anaesthetic agents. The infant also underwent cryopexy for retrolental fibroplasia after topical proparacaine anaesthesia. During the procedure with pressure on the eyeball from the cryoprobe, there were two episodes of bradycardia which resolved with removal of the probe. The infant had no other intraoperative problems and was discharged to the inpatient ward on an apnea monitor. Twelve hours after surgery, the infant was noted to be cyanotic without respiratory effort. No pulse was felt and cardiopulmonary resuscitation instituted. The infant was resuscitated and had a return of normal vital signs. Work-up, including electrolytes, glucose, calcium, blood urea nitrogen, and chest $x$-ray, revealed no abnormalities. The infant had a second episode of apnea after receiving intramuscular pentobarbital for a computed tomography scan of the head. The infant was observed for $48 \mathrm{hr}$ after the second event and had no further problems.

Another issue concerning inguinal herniorrhaphy is at what age this procedure should be performed, especially in the former pre-term infant. While the infant is in the neonatal intensive care unit (NICU), the incidence of incarceration is low. Therefore, it has become common practice to wait until the time of discharge to perform the procedure. Most pediatric surgeons (65\% in the survey performed by Wiener $e t a l .{ }^{12}$ ), because of concerns of incarceration when the infant is discharged home and no longer under close surveillance, advocate this practice.

The two patients that we report had no confounding factors or associated conditions, other than prematurity, which could contribute to perioperative apnea. They had no recent history of apnea, had been discharged home, were not currently on a monitor at home, did not receive supplemental intravenous or inhalational sedative agents, and received only acetaminophen during the postoperative period. Both patients were normothermic and had haematocrit values of 35 and 33\% respectively. Both infants also developed apnea and bradycardia within 5-10 min of the spinal block. In the first patient, the severity of the apnea and periodic breathing seemed to decrease as the spinal block dissipated while pharmacological therapy with caffeine ${ }^{13}$ was administered to the second patient.

Administration of spinal anaesthesia without sedation to a pre-term infant does not eliminate the risk of postoperative apnea and we cannot recommend that spinal anaesthesia be performed on high-risk infants on an outpatient basis. Our clinical experience continues to support the efficacy and safety of spinal anaesthesia in the high risk, former pre-term infant. When they noted no apnea following spinal anaesthesia in 84 high risk infants, Veverka et al. . $^{5}$ suggested that "spinal anaesthesia is safe in an outpatient setting in the high-risk infant". Our findings strongly contradict this conclusion and we suggest that, because the incidence of apnea following spinal anaesthesia is low, a series of 84 infants was not large enough to identify this life-threatening complication. The same monitoring standards should apply to all former pre-term infants considered at risk for apnea regardless of whether they receive general or spinal anaesthesia.

Future studies are needed to define clearly not only the aetiological factors responsible for perioperative apnea, but also the optimal anaesthetic technique for such patients. The majority of the literature supports the notions that: 1) apnea is more likely in those preterm infants with a history of apnea, ${ }^{14}$ 2) supplemental intravenous or inhalational anaesthetic agents increase the incidence of apnea, ${ }^{6}$ and 3 ) following general anaesthesia, caffeine benzoate may decrease the incidence of apnea. ${ }^{13}$ Our two patients demonstrate that apnea can occur even when spinal anaesthesia is used as the sole anaesthetic technique. The mechanisms for this phenomenon remain to be determined.

\section{References}

1 Gregory GA, Steward DJ. Life threatening perioperative apnea in the ex-"premie". Anesthesiology 1983; 59: 495-8.

2 Kurth CD, Spitzer AR, Broennle AM, Downes JJ. Postoperative apnea in preterm infants. Anesthesiology 1987; 66: 483-8.

3 Abajian JC, Mellish RWP, Browne AF, Perkins FM, Lambert DH, Mazuzan JE Jr. Spinal anaesthesia for surgery in the high-risk infant. Anesth Analg 1984; 63: 359-62.

4 Sartorelli $K H$, Abajian JC, Kreutz JM, Vane $D W$. Improved outcome utilizing spinal anaesthesia in highrisk infants. J Pediatr Surg 1992; 27: 1022-5.

5 Veverka TJ, Henry DN, Milroy MJ, et al. Spinal anaesthesia reduces the hazard of apnea in high-risk infants. 
Am Surg 1991; 57: 531-4.

6 Welborn LG, Rice LJ, Hannallah RS, Broadman LM, Ruttiman UE, Fink R. Postoperative apnea in former preterm infants: prospective comparison of spinal and general anaesthesia. Anesthesiology 1990; 72: 838-42.

7 Krane EJ, Haberkern CM, Jacobson LE. Postoperative apnea, bradycardia, and oxygen desaturation in formerly preterm infants: prospective comparison of spinal and general anaesthesia. Anesth Analg 1995; 80: 7-13.

8 Harnik EV, Hoy GR, Potolicchio S, Stewwart DR, Siegelman $R E$. Spinal anaesthesia in premature infants recovering from respiratory distress syndrome. Anesthesiology 1986; 64: 95-9.

9 Webster AC, McKishnie JD, Kenyon CF, Marshall DG. Spinal anaesthesia for inguinal hernia repair in high-risk neonates. Can J Anaesth 1991; 38: 281-6.

10 Cox RG, Goresky GV. Life-threatening apnea following spinal anaesthesia in former premature infants. Anesthesiology 1990; 73: 345-7.

11 Watcha $M F$, Thach BT, Gunter JB. Postoperative apnea after caudal anaesthesia in an ex-premature infant. Anesthesiology 1989; 71: 613-5.

12 Wiener ES, Touloukian RJ, Rodgers BM, et al. Hernia survey of the Section on Surgery of the American Academy of Pediatrics. J Pediatr Surg 1996; 31 : 1166-9.

13 Welborn $L G$, Hannallah $R S$, Fink $R$, Ruttimann UE, Hicks JM. High-dose caffeine suppresses postoperative apnea in former preterm infants. Anesthesiology 1989; 71: 347-9.

14 Welborn LG. Post-operative apnoea in the former preterm infant: a review. Paediatr Anaesth 1992; 2: $37-44$. 